\title{
Radiation Patterns of Multimode Feed-Horn Coupled Bolometers for FAR-IR Space Applications
}

\author{
Eimante Kalinauskaite ${ }^{\mathrm{a}}$, J.Anthony Murphy ${ }^{\mathrm{a}}$, Ian McAuley ${ }^{\mathrm{a}}$, Neil A. Trappe ${ }^{\mathrm{a}}$, Darragh N. \\ McCarthy $^{\mathrm{a}}$, Colm P. Bracken ${ }^{\mathrm{a}}$, Stephen Doherty ${ }^{\mathrm{a}}$, Marcin L. Gradziel ${ }^{\mathrm{a}}$, Creidhe O'Sullivan ${ }^{\mathrm{a}}$, Daniel \\ Wilson $^{a}$, Tully Peacocke ${ }^{\mathrm{a}}$, Bruno Maffei ${ }^{\mathrm{b}}$, Jean-Michel Lamarre ${ }^{\mathrm{c}}$, Peter A. R. Ade ${ }^{\mathrm{d}}$, Giorgio \\ Savini ${ }^{\mathrm{e}}$ \\ ${ }^{a}$ National University of Ireland, Maynooth, Department of Experimental Physics, Maynooth, Ireland; \\ ${ }^{\mathrm{b}}$ The University of Manchester, School of Physics and Astronomy, Manchester, United Kingdom; \\ ${ }^{\mathrm{c}}$ Observatoire de Paris, Paris, France; \\ ${ }^{\mathrm{d} C a r d i f f}$ University, School of Physics and Astronomy, Cardiff, United Kingdom; \\ eUniversity College London, School of Physics and Astronomy, London, United Kingdom;
}

\begin{abstract}
A multimode horn differs from a single mode horn in that it has a larger sized waveguide feeding it. Multimode horns can therefore be utilized as high efficiency feeds for bolometric detectors, providing increased throughput and sensitivity over single mode feeds, while also ensuring good control of the beam pattern characteristics.

Although a cavity mounted bolometer can be modelled as a perfect black body radiator (using reciprocity in order to calculate beam patterns), nevertheless, this is an approximation. In this paper we present how this approach can be improved to actually include the cavity coupled bolometer, now modelled as a thin absorbing film. Generally, this is a big challenge for finite element software, in that the structures are typically electrically large. However, the radiation pattern of multimode horns can be more efficiently simulated using mode matching, typically with smooth-walled waveguide modes as the basis and computing an overall scattering matrix for the horn-waveguide-cavity system.
\end{abstract}

Another issue on the optical efficiency of the detectors is the presence of any free space gaps, through which power can escape. This is best dealt with treating the system as an absorber. Appropriate reflection and transmission matrices can be determined for the cavity using the natural eigenfields of the bolometer cavity system. We discuss how the approach can be applied to proposed terahertz systems, and also present results on how the approach was applied to improve beam pattern predictions on the sky for the multi-mode HFI $857 \mathrm{GHz}$ channel on Planck.

\section{INTRODUCTION}

If the waveguide feeding a horn antenna is oversized then potentially higher order waveguide modes as well as the fundamental mode are capable of coupling signal power to the detector ${ }^{1,2}$. If that detector is a cavity coupled bolometer then these higher order modes essentially ensure increased throughput compared to single mode feeds. One significant advantage of multimode horns is they provide good control of the beam pattern characteristics (as is the case for single mode feed horns) and the feed patterns of systems with standard cross sections can in theory be modelled using efficient mode matching scattering matrix approaches. A disadvantage of course in imaging applications on telescopes is that there is necessarily reduced spatial resolution (assuming the multimode horn throughput is matched to the optics coupled to it). This is because the throughput, given by $A \Omega=n \lambda^{2}$ where $n$ is the number of modes present, is higher than for the single mode case thus leading to larger beams on the sky.

In many cases it may be a reasonable approximation to assume that the cavity mounted bolometer acts as a perfect black body absorber ${ }^{1,2}$. Thus, for the purposes of modelling the system to obtain the horn beam patterns, we can apply reciprocity and assume that all modes are equally excited in the waveguide that directly feeds the bolometer cavity. Clearly, this is an approximation, particularly because of any impedance mismatches the modes may suffer at the entrance to the cavity (if

Terahertz, RF, Millimeter, and Submillimeter-Wave Technology and Applications X, edited by

Laurence P. Sadwick, Tianxin Yang, Proc. of SPIE Vol. 10103, 101030N · @ 2017 SPIE CCC code: $0277-786 \mathrm{X} / 17 / \$ 18 \cdot$ doi: $10.1117 / 12.2249869$

Proc. of SPIE Vol. 10103 101030N-1 
there is a sudden step in the diameter of the structure), and furthermore the individual modes in the cavity may not perfectly couple to the bolometer/absorber. Thus, in certain cases the perfect blackbody assumption about feeding the radiating hornwaveguide structure could become a poor one, and a more thorough approach to modelling the system is clearly required. Such horn-waveguide-cavity configurations could be modelled using finite element methods, but multimode structures by their nature are electrically large, and therefore any simulations will be computationally intensive. On the other hand, one expects that a modal approach by its nature (i.e. using solutions to the propagating electromagnetic fields based on the symmetries of the system) should be more efficient. In this paper we report on some of the work we have undertaken to investigate cavity effects on the signal coupling to the bolometer for particular examples where a finite element analysis would have been very difficult.

One improvement to the accuracy of such mode matching simulations is to model the bolometer as a thin current sheet of finite surface resistance, in which lossy currents are induced by the fields at its surface ${ }^{3,4,5}$. Then, any real power that is not reflected back into propagating modes in the horn plus waveguide feeding the cavity must have been converted into Joule heating of the effective power absorbing sheet. Thus, the beam pattern of the horn can be determined from the strength of the coupling of the incident radiation from a point in the sky with those modes that are efficiently coupled to the absorbing cavity. Note that in this case we have not applied reciprocity, and instead treat the horn aperture as the input port to the full system. This approach however assumes no other loss of power, such as due to any gaps in the effective perfectly conducting walls of the feed system of the cavity. Such gaps could be present for the insertion of filters, for example, or in the case of an array system where a gap is necessary to prevent shorting of the detectors which are fabricated on a chip that necessarily straddles several detector elements. In such cases the power reflected at the horn aperture will be reduced because of extra losses due to radiation escaping in such gaps. Furthermore, because of reflections within the system we cannot easily disentangle the power absorbed in the cavity from such power losses through any gaps. In fact we are left with an assumption that it is better in fact to invoke reciprocity as before, treating the cavity as an imperfect black body radiator. In this paper we describe an alternative approach based on the natural eigenfields of the absorbing cavity and some simple thermodynamic assumptions.

In Section 2 we review the theory for modelling absorbing cavities and show how this can be extended for the purposes of reciprocity when dealing with lossy feed systems. In Section 3 we further discuss an example of a cavity mounted absorbing detector with a lossy free space gap behind the horn that effectively forms the beam, so that radiation escapes at some detectable level before being detected. This example is based on the $857 \mathrm{GHz}$ multi-mode pixels of the HFI instrument on the European Space Agency Planck satellite and we compare our prediction with some pre-launch laboratory measurements. We also show how the improved Planck multimode beams on the sky based on this analysis compared with beam pattern measurements made on planets as reported in the literature. In Section 4 we draw some conclusions from the work.

\section{ABSORBER CAVITIES}

In the case of an absorbing cavity we make the approximation that the absorber acts as a resistive current sheet, with the resulting Joule heating being detected by utilising a temperature dependent characteristic of the device (e.g. TES detectors $)^{3,45}$. The difference in the tangential magnetic fields at the two surfaces (left and right) of the absorber are related

to the current flowing in the current sheet by $\mathbf{H}_{L}=\mathbf{H}_{R}-\mathbf{K} \times \hat{k}$ (where $\hat{k}$ is a unit vector in the direction of propagation and $\mathbf{K}$ is the surface current density). This current is driven by the finite tangential electric field present because of the surface resistance due to the finite conductivity of the material of the thin sheet: $\mathbf{K}=\mathbf{E}_{L} / R_{S}=\mathbf{E}_{R} / R_{S}$. Thus, relationships exist between the electric and magnetic fields, which couple them together at the plane of the sheet causing an average flow of energy into the absorber as well some scattering of non-absorbed power between the modes if the absorber does not fill the full diameter of the waveguide. These relationships are also affected by the guide impedance appropriate for the modes at the absorber.

This theory has been described previously ${ }^{2,3,6}$ and we summarise the main points here. The resulting scattering matrices for the case when the absorber is partially, or completely, filling the surrounding waveguide (or cavity) cross-section can be derived and are given by: 


$$
\begin{gathered}
S_{11}=-\left[Q^{*}+\frac{Z \cdot T}{2 R_{S}}\right]^{-1} \cdot \frac{Z \cdot T}{2 R_{S}}=S_{22} \\
S_{12}=-\left[Q^{*}+\frac{Z \cdot T}{2 R_{S}}\right]^{-1} \cdot Q^{*}=S_{21}
\end{gathered}
$$

where $S_{11}, S_{12}, S_{21}$ and $S_{22}$ represent the usual scattering matrices at a discontinuity, $Z$ is a diagonal matrix of mode impedances, $Q$ is the self-coupling of a mode just outside the absorber (usually normalised to unity for propagating modes and $\pm i$ for evanescent modes, depending on whether they are TE or TM) and $T$ is a matrix of coupling integrals of the form $T_{i j}=\int \mathbf{e}_{i}^{L} \cdot \mathbf{e}_{j}^{R^{*}}$. $\mathbf{d S}$ over the absorber surface. These matrices can then be cascaded with the scattering matrices for the rest of the structure in the usual manner for waveguides.

In this case if we calculate the reflection scattering matrix $S_{11}$ for the system as a whole including the horn antenna, waveguide feed and cavity, where again we can infer that power that is not reflected back into backward propagating modes at the horn aperture must have been absorbed by the cavity. Care must be taken with evanescent modes in this approach.

As mentioned earlier using mode matching, the power absorbed can be extracted from the overall $S_{11}$ matrix for the complete horn-waveguide-absorbing bolometer system treating the horn aperture as the input port (and not applying reciprocity). We can then infer that power that is not reflected back into backward propagating modes at the horn aperture must have been absorbed by the cavity. In doing this the cavity structure is essentially the last section to be cascaded and it is regarded a waveguide structure with one end closed and taking into account that power is lost due to absorption by the current sheet. With this approach it is then possible to recover the absorbed power for each propagating $n$ at the horn aperture (input port) that is coupled to the incident field from the sky (a plane wave at the antenna generated by a point source at infinity in the direction $(\theta, \varphi)$ ). Thus, the total power absorbed for mode $n$ can be derived from the average reflected power back into the modes propagating in the backward direction (average loss of power propagating backwards implies absorption in the cavity). This can be calculated from the $S_{11}$ matrix at the horn aperture and the beam on the sky $P_{\text {sky }}(\theta, \varphi){ }^{4}$

However, as discussed in the introduction, this approach cannot be used if there is another source of loss in the system such as a free space gap through which signal power can escape, since it is not possible to distinguish between the power absorbed in the cavity and power lost through any gaps in the system. Thus, we model the full bolometer system by splitting the system into two different parts: (i) the absorbing cavity plus its feed waveguide and (ii) the rest of the system including any feed horn and waveguide sections with gaps, and also apply reciprocity as before in contrast to the approach above where the horn aperture is the input port. The overall $S_{21}$ matrix of the whole system (required to determine the beam pattern of the horn using reciprocity) is obtained by cascading the resulting matrices for both sub-sections: $S_{21}^{\text {full }}=$ $S_{21}^{\text {horn }} \cdot\left[I-S_{22}^{\text {cavity }} \cdot S_{11}^{\text {horn }}\right]^{-1} \cdot S_{21}^{\text {cavity }}$, where the cavity clearly refers to cavity and horn refers to the rest of the system including the horn antenna and the waveguide structure with the lossy gap. This equation ${ }^{7}$ essentially indicates that in order to model the absorbed power and include both the lossy sections in the detector element (free-space gap) and the bolometer in the cavity, the cavity needs to be modelled as a radiator with an appropriate $S_{21}$ derived.

We can construct an approximation to the $S_{21}^{\text {Cavity }}$ using an SVD approach. Beginning with, singular value decomposition (SVD) applied to reflection from the cavity: $S_{22}=U \cdot \Sigma_{22} \cdot V^{+}$, where $\Sigma_{22}$ is a diagonal matrix of singular values $\left[\sigma_{1}, \sigma_{2}\right.$, $\left.\sigma_{3}, \ldots ..\right]$, and $U$ and $V$ are the left and right singular vectors of $S_{22}$. The resulting $S_{21}$ matrix is then constructed using $S_{21}=$ $U . \Sigma_{21} \cdot V^{+}$, where $\Sigma_{21}$ is a diagonal matrix of singular values $\left[\sqrt{1-\sigma_{1}^{2}}, \sqrt{1-\sigma_{2}^{2}}, \sqrt{1-\sigma_{3}^{2}}, \ldots \ldots.\right]$. 
In the next section we consider how this can be applied to the Planck $^{8} 857 \mathrm{GHz}$ pixels.

\section{PLANCK 857 GHZ PIXEL}

The simulation tool required for this analysis is based on in-house mode matching software written in Python (cylindrical version for axially symmetric structures, including horns and cavities) to be able to model absorbers and free space gaps, through which power can escape. The $857 \mathrm{GHz}$ pixel, the highest frequency channel of the HFI instrument on the European Space Agency Planck statellite, is highly overmoded ${ }^{8,9,10}$. The Planck space observatory mapped the anisotropies of the cosmic microwave background with unprecedented sensitivity and angular resolution ${ }^{11}$. The highest frequency channels were required for removal of foreground sources. Figure 3.1 shows a schematic of the back-to-back horn arrangement coupled to the detector horn which fed a spider-web bolometer. Thermal filters were used to thermally insulate the detector horn and bolometer. Simulations of the beam patterns of the $857 \mathrm{GHz}$ pixel were performed across the frequency band ( $729 \mathrm{GHz}$ to $986 \mathrm{GHz})$.

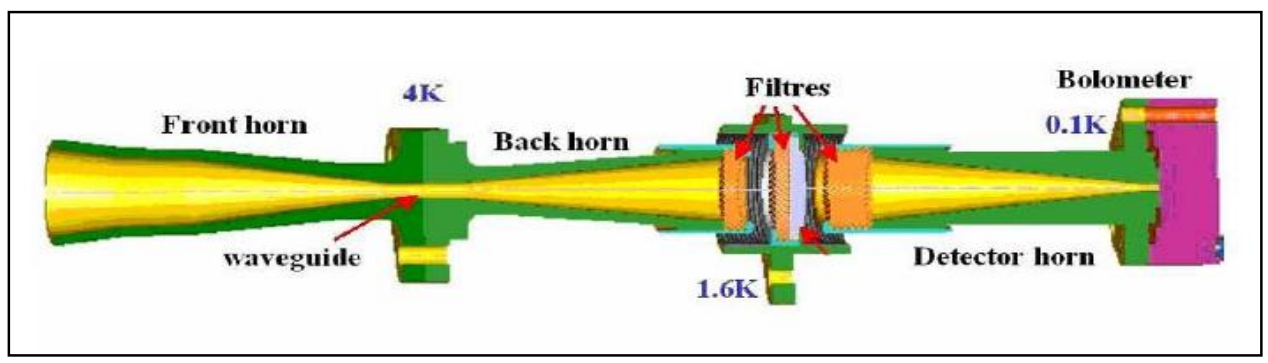

Figure 3.1: The $857 \mathrm{GHz}$ pixel

In the following sections we will discuss the simulations of the beam patterns of the $857 \mathrm{GHz}$ channel that were performed in order to determine the shape of the beam.

\subsection{Beam patterns of $857 \mathrm{GHz}$ pixel as a perfect black body radiator and continuous waveguide at filter gap}

Figure 3.2 shows a plot of the geometry file of the $857 \mathrm{GHz}$ pixel consisting of a back-to-back horn pair, detector feed horn and detector cavity (it is opposite in orientation to Figure 3.1). The thick lines indicate that the waveguide was corrugated except for the thermal filter section. The waveguide in the thermal filter section approximates the metal sleeve over the filters as indicated in Figure 3.1. In this section we first report on treating the horn without the free-space gap so that the filter section (between the back horn and the detector horn) is still a continuous waveguide and the cavity was assumed to be a perfect blackbody absorber. Thus, in this case we assume that all waveguide modes are excited in the waveguide at the entrance to the cavity. We can then compare this "ideal" horn-cavity arrangement with the case where the right and left walls of the filter section are missing, forming two gaps through which power can leak. 


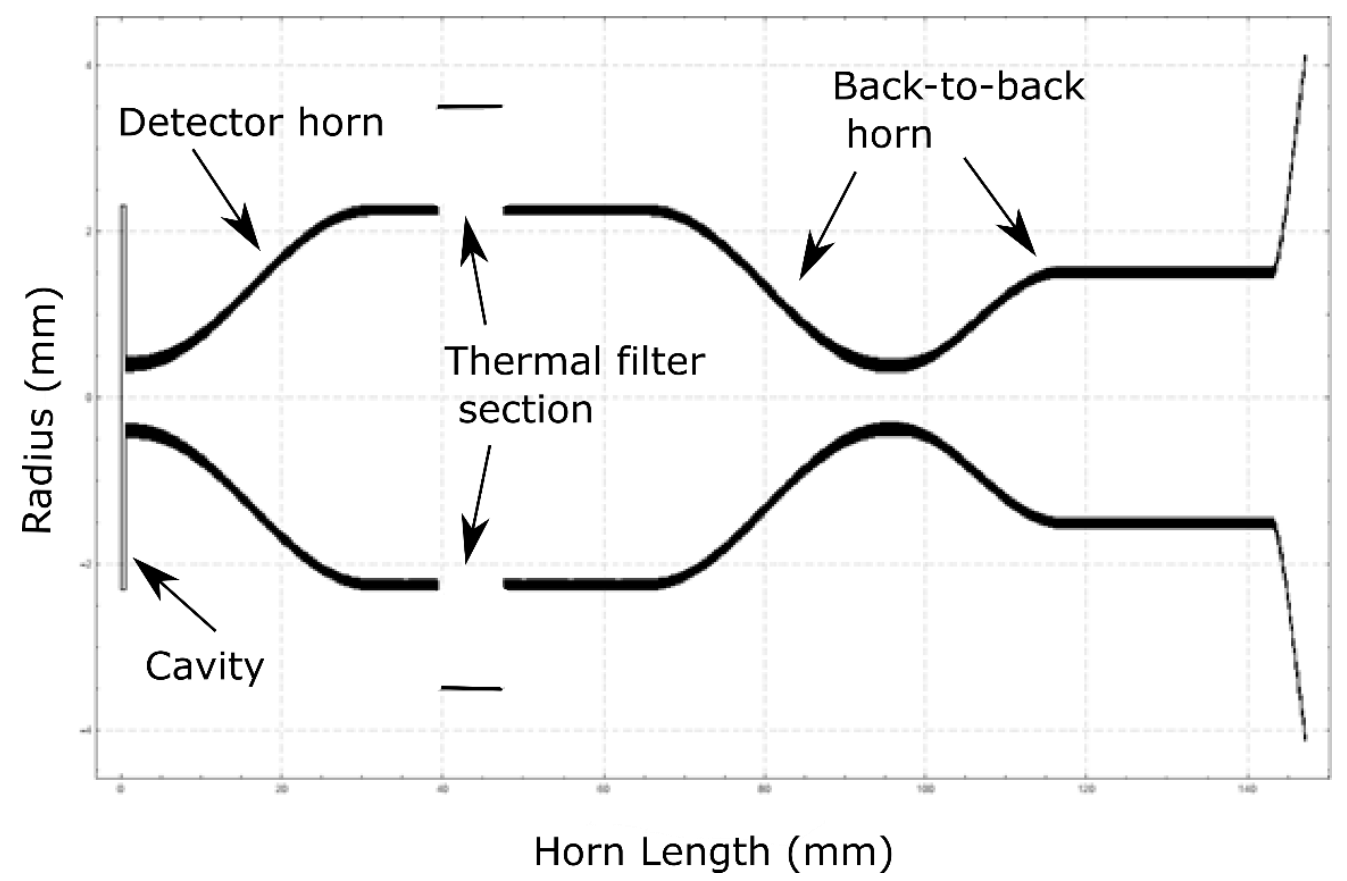

Figure 3.2: Geometry plot of the $857 \mathrm{GHz}$ horn

Figure 3.3 shows the power pattern at the centre of the operating band $(857 \mathrm{GHz} \pm 15 \%)$ as predicted by mode matching software.

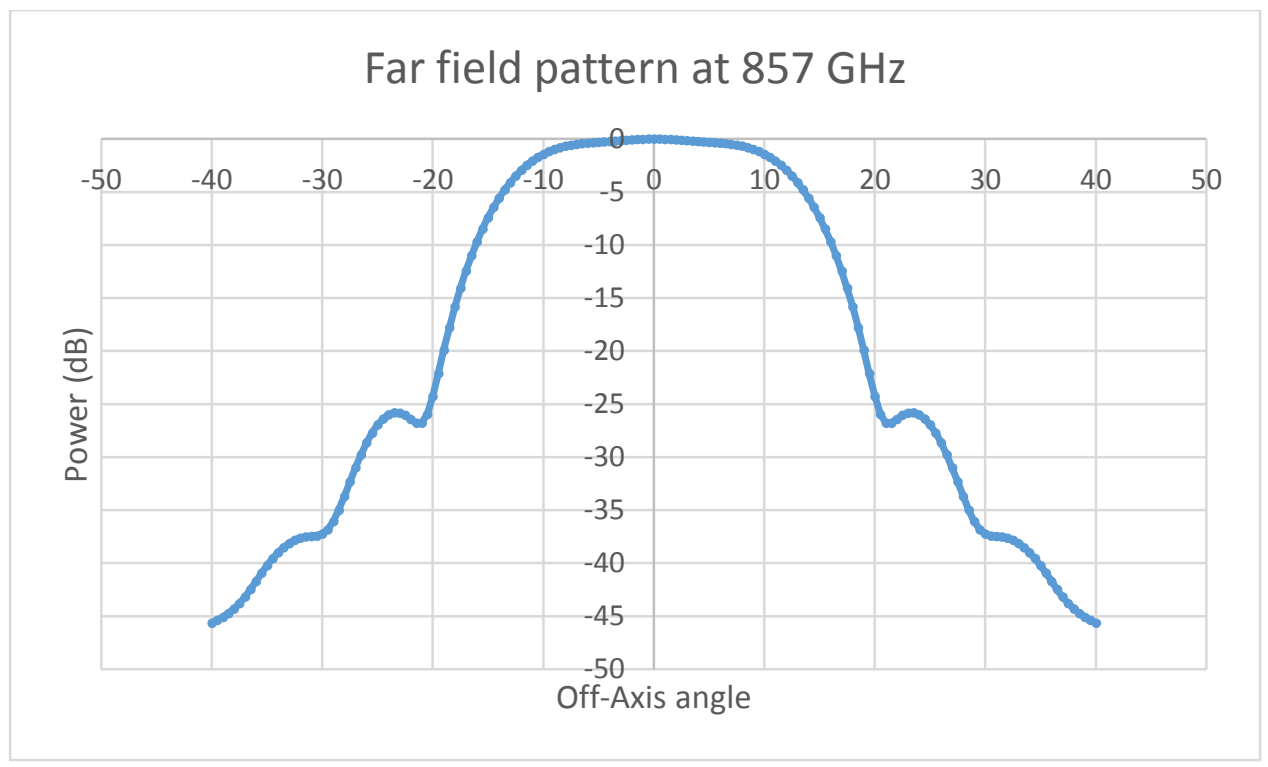

Figure 3.3: Simulations of the far field power pattern of the $857 \mathrm{GHz}$ channel at $857 \mathrm{GHz}$ using mode matching. The thermal filter gap was implemented as a continuous waveguide section and the cavity considered was a perfect blackbody absorber 


\subsection{Beam patterns of $857 \mathrm{GHz}$ pixel as a perfect black body radiator including losses at thermal filter gap}

As can be seen in Figure 3.2, the thermal filter section is not a continuous waveguide, meaning that power carried by the individual modes is lost through the gaps on the right and left where it meets the detector horn and the back horn. Thus the normal scattering matrices that are used to describe scattering at a waveguide section cannot be used in this case. In fact, the appropriate scattering matrices for the waveguide to filter through the free-space section can be approximated as: $S_{11}=$ $S_{22}=[0]$ and $S_{12}=S_{21}{ }^{\dagger}=P$, where $P$ represents the power coupling between the smaller and the larger radius waveguide while the scattering matrices for a free-space to waveguide section are almost the same except $S_{21}=S_{12}^{\dagger}=P$. The power losses due to the gap were investigated by using a perfect blackbody cavity and extracting the singular values immediately before and after the gap. A reduction in the singular values points to loss of power in the resultant hybrid modes in the back horn after propagation through the filter section. The results can be seen in table 3.1.

\begin{tabular}{|c|c|c|c|c|c|c|c|c|c|c|}
\hline \multicolumn{1}{|c|}{ Azimuthal order and mode number (at 857 GHz) } \\
\hline & $\mathbf{0 1}$ & $\mathbf{0 2}$ & $\mathbf{0 3}$ & $\mathbf{1 1}$ & $\mathbf{1 2}$ & $\mathbf{1 3}$ & $\mathbf{2 1}$ & $\mathbf{2 2}$ & $\mathbf{3 1}$ & $\mathbf{3 2}$ \\
\hline Singular value before gap & 1 & 1 & 0.999 & 1 & 1 & 1 & 1 & 1 & 1 & 1 \\
\hline Singular value after gap & 0.896 & 0.844 & 0.620 & 1 & 0.730 & 0.515 & 0.739 & 0.970 & 0.481 & 0.46 \\
\hline
\end{tabular}

Table 3.1: Singular values immediately before and after the thermal filter gap

Figure 3.4 shows the power pattern at $857 \mathrm{GHz}$ as predicted by mode matching software with the cavity modelled as a perfect blackbody absorber and the losses associated with the thermal filter gap now accounted for.

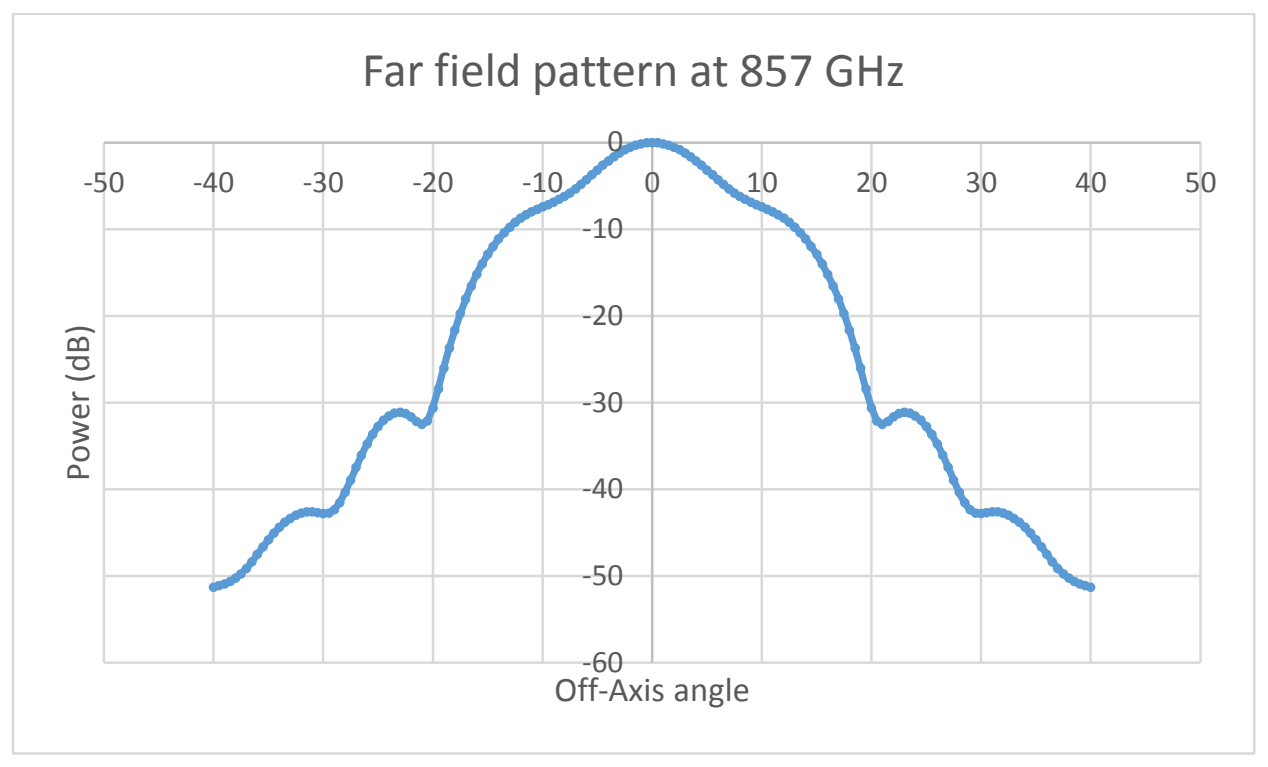

Figure 3.4: Simulations of the far field power pattern of the $857 \mathrm{GHz}$ channel using mode matching. The free-space gap was implemented and the cavity considered was a perfect blackbody absorber

As can be seen from figures 3.3 and 3.4, including the losses at the filter gap has a significant effect on the resulting beam pattern. 


\subsection{Beam patterns of $857 \mathrm{GHz}$ pixel including cavity coupled bolometer and losses and thermal filter gap}

In this section we show the resulting beam patterns when both the free-space gap and the bolometer in the cavity were modelled. Up until this point, the cavity was modelled as a perfect blackbody absorber. Now including a description of the absorber in the cavity we can compute the actual reflection and transmission matrices for the cavity as a radiator $\left(S_{21}\right.$ and $S_{22}$ ) using the SVD approach outlined in section 2 of this paper

Table 3.2 shows the singular modes extracted at the aperture of the horn at the central frequency of the band $(857 \mathrm{GHz})$. The singular values are compared for the case where (i) no losses were modelled in the structure (thermal filter gap treated as a continuous waveguide junction and cavity modelled as a perfect blackbody absorber), (ii) the losses due to the filter gap were included and the cavity was modelled as a perfect blackbody absorber, and (iii) the losses due to the filter gap were included and the bolometer was modelled within the cavity. It can be seen in table 3.2 and figure 3.5 that the freespace gap at the thermal filter section has a significant effect on the resulting beam pattern.

\begin{tabular}{|c|c|c|c|c|c|c|c|c|c|}
\hline \multicolumn{7}{|c|}{ Azimuthal order and mode number (857 GHz) } \\
\hline & $\mathbf{0 1}$ & $\mathbf{0 2}$ & $\mathbf{1 1}$ & $\mathbf{1 2}$ & $\mathbf{1 3}$ & $\mathbf{2 1}$ & $\mathbf{2 2}$ & $\mathbf{3 1}$ & $\mathbf{4 1}$ \\
\hline $\begin{array}{c}\text { Singular Values (Ideal } \\
\text { Case) }\end{array}$ & 1 & 1 & 1 & 0.993 & 0.95 & 1 & 0.955 & 0.889 & 0.811 \\
\hline $\begin{array}{c}\text { Singular Values (Gap) } \\
\begin{array}{c}\text { Singular Values (Gap and } \\
\text { Bolometer) }\end{array}\end{array}$ & 0.762 & 0.301 & 0.986 & 0.526 & 0.411 & 0.516 & 0.197 & 0.201 & 0.25 \\
\hline
\end{tabular}

Table 3.2: Singular values of propagating modes at $857 \mathrm{GHz}$

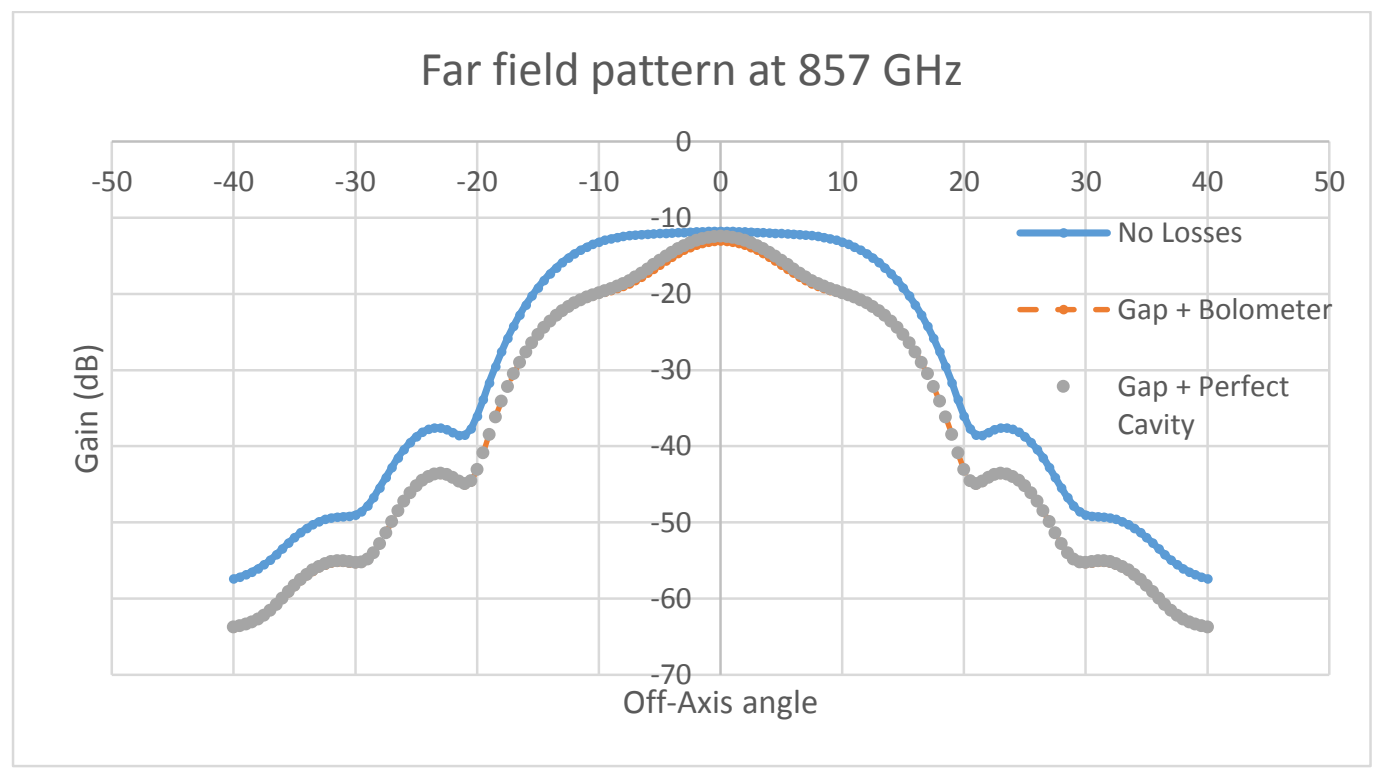

Figure 3.5: Beam patterns comparing the case where no losses are present (continuous line) in the structure with the case where the free-space gap was modelled (dotted line) and also where both the free-space gap and bolometer inside the cavity were modelled (dashed line) 
From figure 3.5, it is clear that the free space gap has a significant effect both on the shape of the beam and the overall throughput of the system. It is also clear that the bolometric cavity of the $857 \mathrm{GHz}$ is very close to being a perfect absorber resulting in minor differences in both throughput and beam pattern between the two cases (perfect black body cavity plus gap and non-perfect absorbing cavity with free space gap).

\subsection{Comparison of simulated and measurement results for far field patterns of the $857 \mathrm{GHz}$ pixels}

Broad band beam pattern simulations were carried out on the $857 \mathrm{GHz}$ Planck pixel using mode matching. The simulations were then compared to broad band frequency measurements of the horn far field made at Cardiff University ${ }^{1}$. The comparison can be seen in figure 3.6.

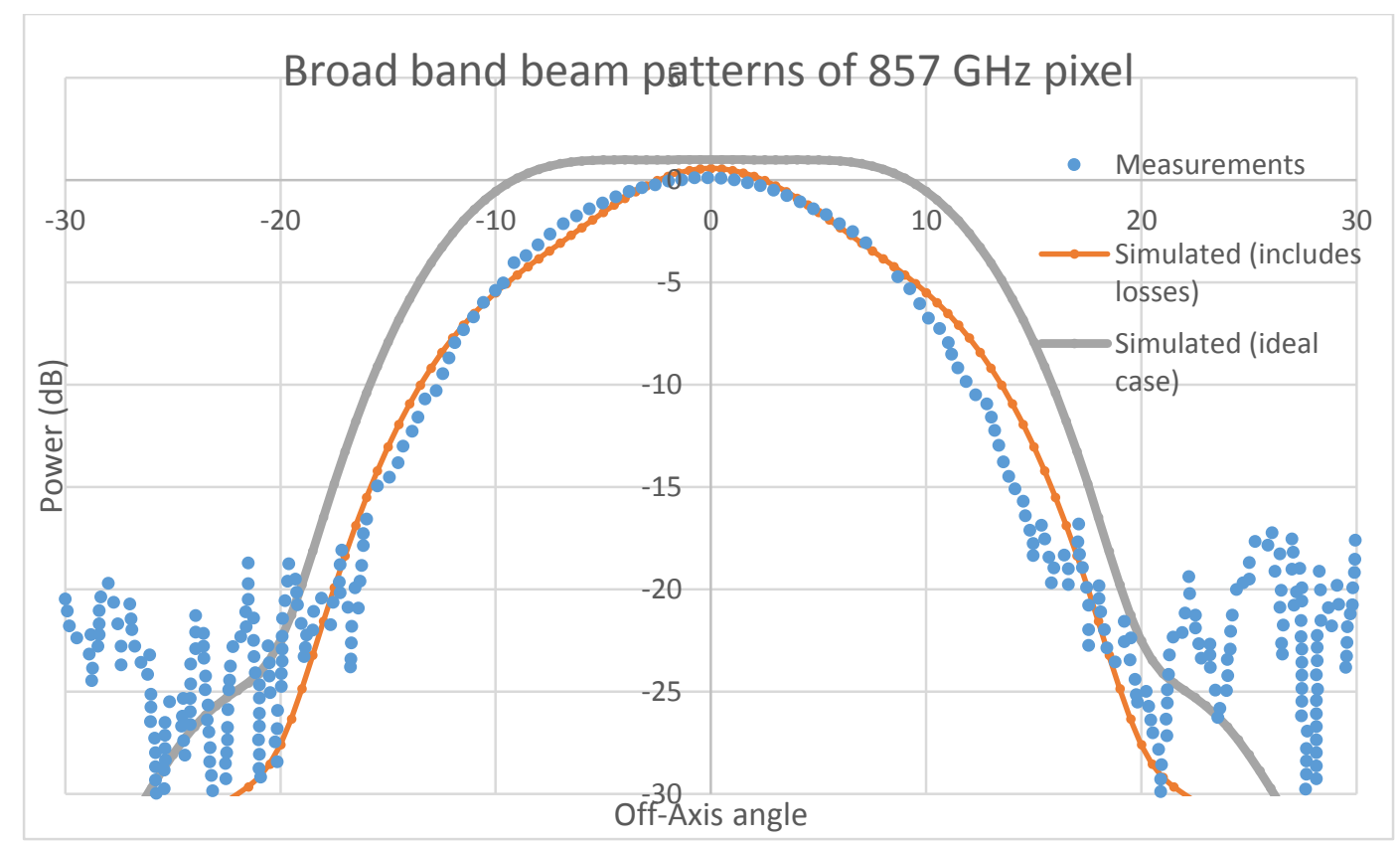

Figure 3.6: Measured and simulated broadband beam patterns of the $857 \mathrm{GHz}$ pixel

It is clear that the losses in the pixel need to be accounted for in order to get reasonable agreement between simulations and measurements. In figure 3.6, the data points refer to the broadband beam measurements carried out in Cardiff University ${ }^{1}$, the thin curve refers to the broadband beam pattern simulations for the case where both the free-space gap and the bolometer were modelled inside the cavity. The thick curve in figure 3.6 represents the broadband beam pattern of the pixel for the case where no losses were present (the free-space gap was treated as a continuous waveguide section and the bolometer was a perfect blackbody absorber). It is clear that the agreement is much better when the losses within the pixel are accounted for.

\subsection{Modelling of the Planck $857 \mathrm{GHz}$ beam patterns on the sky}

The aperture field patterns of the modes supported by the $857 \mathrm{GHz}$ horn were obtained using mode matching software by obtaining the singular values of the overall $S_{21}$ matrix of the pixel and then multiplying the singular value entries by the left singular vectors of the $S_{21}$ matrix. The beams on the sky were obtained using physical optics software and propagating the individual singular vectors through the telescope and onto the sky. These beams on the sky were then added in quadrature. Figure 3.7 shows a comparison between the resulting beam on the sky at $857 \mathrm{GHz}$ between the ideal system (where the thermal filter gap was treated as a continuous waveguide junction and the cavity was a perfect blackbody 
absorber) and the lossy system (where the losses at the thermal filter gap were accounted for and the bolometer was modelled inside the cavity).

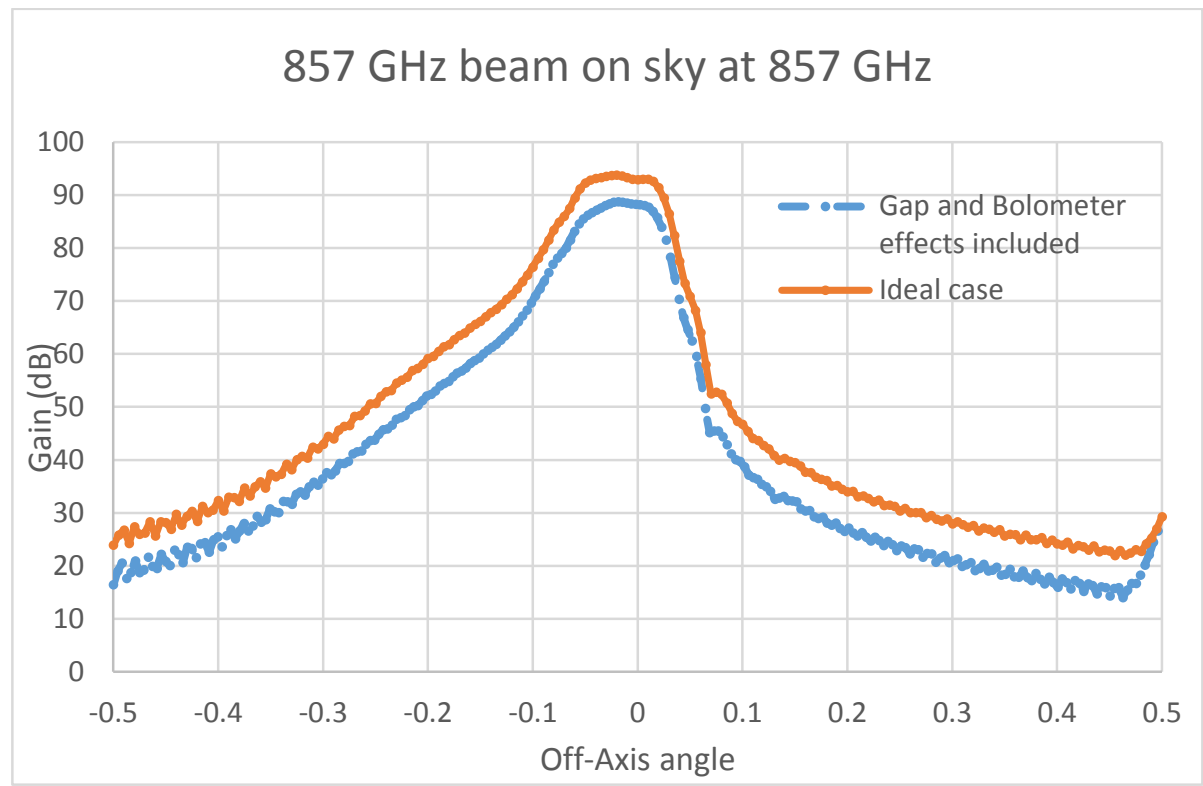

Figure 3.7: Resulting beam on the sky modelled at $857 \mathrm{GHz}$ for the case where no losses were considered (continuous line) and for the case where a bolometric cavity and free-space gap were modelled (dashed line)

It is clear from figure 3.7 that the beam on the sky is less top hat like when the losses within the horn structure are accounted for (which is also the case for the predicted far field beam patterns of the horn). Figure 3.8 shows the comparison of the simulated data with averaged main beam profile derived from observations of planets (Planck collaboration, 2014) ${ }^{12}$

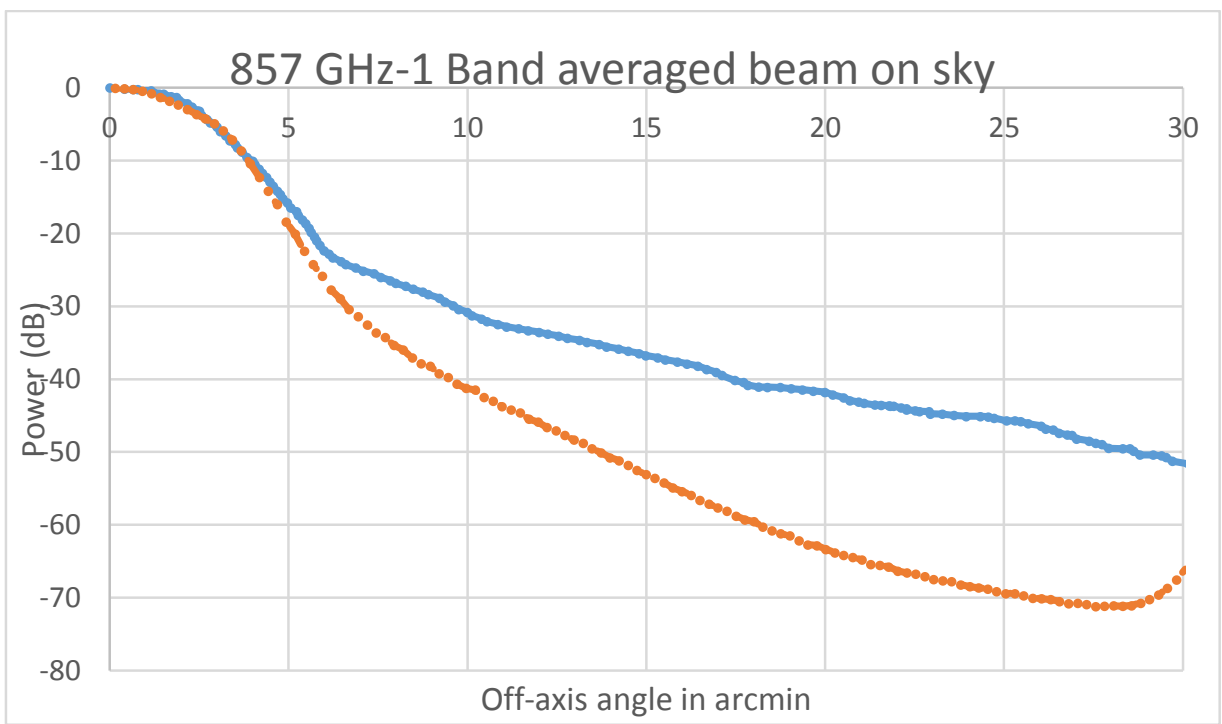

Figure 3.8: Band averaged main beam profiles comparing the mode matching and PO model (dashed line) with main beam profile derived from observations of planets (continuous line) 


\section{CONCLUSIONS}

In this paper we have presented how mode matching may be used to efficiently model feed horn coupled bolometers. Inhouse mode matching software written in Python was used to model multimode cavity coupled bolometers (such as the Planck $857 \mathrm{GHz}$ pixel). The software was extended to include free-space gaps in the structure, which made significant improvements on the predicted beam pattern of the HFI $857 \mathrm{GHz}$ channel on Planck. Agreement with both pre-launch laboratory measurements and with the beams on the sky indicated that some power was in fact lost at the filter section although still operated as a multimode detector.

\section{ACKNOWLEDGEMENTS}

This research has been funded as part of a research contract with the European Space agency under the Strategic Initiative for Irish Industry, Academia and Research Institutes (2012 - 2017).

\section{REFERENCES}

[1] Murphy, J. A. et al, (2010). "Multi-mode horn design and beam characteristics for the Planck satellite", J. Inst. Journal of Instrumentation, $5(04)$.

[2] Murphy, J. A. et al, (2001). "Radiation patterns of multi-mode corrugated horns for far-IR space applications", Infrared Phys. Technol. 42 (2001) 515

[3] Murphy, J. A. et al, (2012). "New developments in waveguide mode-matching techniques for far-infrared astronomy", SPIE 8261, Terahertz Technology and Applications V, 82610F (2 March 2012); doi: $10.1117 / 12.908068$.

[4] Doherty, S., "Optical and Quasi-Optical Design and Analysis of Astronomical Instrumentation including a Prototype SAFARI Pixel", PhD thesis, National University of Ireland Maynooth, 2012.

[5] Bracken, C., "Electromagnetic Techniques for Analysis and Design of Ultra Sensitive Receivers for Far-Infrared Astronomy”, Ph.D. thesis, NUI Maynooth, 2015

[6] Kalinauskaite, E. et al, (2016). "Modelling multimode feed-horn coupled bolometers for millimeter-wave and terahertz astronomical instrumentation", SPIE 9914, Millimeter, Submillimeter, and Far-Infrared Detectors and Instrumentation for Astronomy VIII, 99142R (July 19, 2016); doi:10.1117/12.2231444.

[7] Olver, A.D., Clarricoats, P., Kishk, A., and Shafai, L., "Microwave Horns and Feeds", IEEE Press, New York, 1994.

[8] Tauber, J.A. et al, (2010). "Pre-launch status of the Planck mission", Astronomy \& Astrophysics, 520.

[9] Ade, P. A. R. et al, "Planck pre-launch status: The optical architecture of the HFI", Astronomy \& Astrophysics, 520, A11

[10] Maffei, B. et al, (2010). "Planck pre-launch status: HFI beam expectations from the optical optimisation of the focal plane", Astronomy and Astrophysics A\&A,520.

[11]Planck Collaboration: Ade, P. A. R. et al, (2014). "Planck 2013 results. I. Overview of products and scientific results", Astronomy \& Astrophysics, $571 \mathrm{~A} 1$.

[12] Planck Collaboration: Ade, P. A. R. et al, (2014). "Planck 2013 results. VII. HFI time response and beams", Astronomy and Astrophysics A\&A, 571 A7. 